\title{
Advances in Electron-Probe Microanalysis and Compositional Mapping: Applications to the Analysis of Meteorites
}

\author{
P. K. Carpenter ${ }^{*}$, R. A. Zeigler , B. L. Jolliff*, E. P. Vicenzi ${ }^{* *}$, J. M. Davis ${ }^{* * *}$, C. M. MacRae ${ }^{* * * *}$, N. C. Wil- \\ $\operatorname{son}^{* * * *}$, P. G. Kotula ${ }^{* * * * *}$, and J. J. Donovan ${ }^{* *}$ \\ "Dept. Earth and Planetary Sciences and the McDonnell Center for the Space Sciences, Washington Univer- \\ sity in St. Louis, Campus Box 1169, St. Louis, MO, 63130 \\ ${ }^{* *}$ Smithsonian Institution, Museum Conservation Institute, Suitland, MD 20746 \\ **** Microanalysis Research Group, National Institute of Standards and Technology, Gaithersburg, MD 20899 \\ ${ }^{* * * *}$ CSIRO Minerals, Bayview Ave., Clayton, VIC 3168, Australia \\ ***** Sandia National Laboratories, PO Box 5800, Albuquerque, NM 87185-0886 \\ ******* Camcor, 1241 University of Oregon, Eugene, OR 97403.
}

Improvements in EPMA and $\mu \mathrm{XRF}$ instrumentation has enabled large-scale X-ray mapping of meteorite samples. The advent of silicon-drift (SDD) energy-dispersive (EDS) detectors with high-throughput digital processing electronics has dramatically decreased mapping acquisition times and increased sensitivity. EPMA provides a relatively high spatial resolution of $\sim 1 \mu \mathrm{m}$ and allows sampling of up to 50-100 $\mu \mathrm{m}$ spot in defocused-beam analysis (DBA), and can analyze a wide range of elements including low energy X-rays, but trace element detection is ultimately limited by continuum X-ray production. Analysis by $\mu \mathrm{XRF}$ typically uses a minimum spot size of 50-100 $\mu \mathrm{m}$, has superior trace element sensitivity and can be used on materials which are not vacuum compatible or exhibit electron-beam damage in the microprobe, but requires thicker samples for analysis due to X-ray penetration and has relatively poor low energy X-ray sensitivity. The two techniques are complimentary for these reasons.

Compositional mapping typically makes use of backscattered-electron mosaic base maps which are correlated with X-ray maps acquired using stage scan mode. Region-of-interest (ROI) and spectrum image X-ray data sets acquired for samples containing in excess of ten elements require significant storage and data processing capabilities. We have analyzed samples using several software packages and will compare both qualitative and quantitative mineral classification and analysis using these tools.

EPMA has seen improvements in $\Phi(\rho z)$ algorithms and more accurate sets of mass absorption coefficients have improved quantitative analysis by both techniques. In quantitative analysis, the calculated concentration $\mathrm{C}$ is determined by iterative calculation from $\mathrm{C}=\mathrm{k} * \mathrm{ZAF}$, which implicitly requires knowledge of the concentration of all elements in the sample volume and their effect on the element of interest. The Bence-Albee $\alpha$-factor algorithm originally used constant term $\alpha$-factors, the accuracy of which is known to be deficient, and a polynomial formulation has been used to generate $\alpha$-factors which duplicate $\Phi(\rho z)$ algorithms and can be used for simple and rapid correction of simulated analysis problems and X-ray compositional map data $[1]$.

Mapping and spot analysis of multiphase materials results in excitation of a volume containing multiple phases, and accurate analysis must correct for X-ray generation and emission from discrete mineral phases rather than treating the volume as a homogeneous single phase. With reference to analysis of homogeneous materials, it is necessary to know the phase composition for iterative correction, and the phase density for conversion from sampled area fraction to weight fraction of the phase. Omission of a density conversion introduces significant errors in the analysis [2]. In DBA the contribution from each discrete phase is included as a weight fraction term in the iteration loop, and requires either explicit knowledge of the mineral chemistry or an approximation using a CIPW normative mineral calculation. The DBA technique and errors inherent in the calculation have been discussed previously [3-7]. 
Several problems exist in the general analysis of multiphase volumes. For phase mixtures with a grain size larger than the electron scattering volume (i.e., larger than $10 \mu \mathrm{m}$ ) the treatment of discrete phases is essentially identical for EPMA and $\mu$ XRF because the sample must be deconvolved into the constituent phases for correction. The role of electron scattering from one phase to another is dimensionally secondary. For mixtures with a grain size that is smaller than the scattering volume (i.e., less than $\sim 1 \mu \mathrm{m}$ ), or for grain geometries that include inclined and shallow boundaries, the role of differential electron backscattering is more important. In the case of $\mu \mathrm{XRF}$ analysis, the primary fluorescing X-rays penetrate between 30 and $100 \mu \mathrm{m}$ before becoming fully attenuated, and can produce lateral sampling areas as much as twice the incident beam area. Current research has focused primarily on characterizing macro phases larger than the incident beam. Such methods require quantification and refinement using the fundamental parameters algorithm [8].

To date, no systematic analysis of physical mixtures of microanalysis standards has been conducted. The accuracy of DBA analysis on lunar materials was confirmed by comparison to bulk analysis which clearly did not utilize exactly the same material. A polynomial $\alpha$-factor formulation has been utilized in an Excel VBA interface designed to simulate X-ray production and emission from multiphase samples in support of DBA data correction. An sample comprised of equal area fractions of anorthite and an $\mathrm{En}_{80} \mathrm{Fs}_{20}$ pyroxene is shown in Table 1. The ideal weight percent analysis was calculated from the known area fraction and density values for the two phases. The k-ratios for anorthite and the pyroxene were assumed to vary linearly with area and were input in the DBA correction using the ZAF factors for anorthite and pyroxene. The k-ratio data was also used to calculate an analysis for an assumed homogeneous sample. Disagreement of both the DBA and homogeneous correction with the ideal analysis suggest that the assumption of linear X-ray emission with area may not be fully justified, and that the densities used for calculation of the ideal analysis are not compatible with the atomic number component of the ZAF correction. These and other software tools for simulation of electron scattering and X-ray production via Monte Carlo and $\Phi(\rho z)$ algorithms used for bulk and thin-film sample geometries allow for complimentary analysis of multiphase samples. We are applying these tools to the analysis of lunar and martian meteorites as part of ongoing research that combines microanalysis with an evaluation of local and overall bulk chemistry and mineralogy [9-10].

References

[1] Armstrong, J. T. (1988) Microbeam Analysis, 469-476.

[2] Warren, P. (1997) LPS XXVIII, 1497-1498.

[3] Albee, A. L. et al. (1977) $8^{\text {th }}$ Int. Congr. Xray Opt. 526-537.

[4] Albee, A. L. et al. (1977) LPS VIII, 7-9.

[5] Nazarov, M. A. et al. (1982) LPS XIII, 582-583.

[6] Lindstrom, D. J. (1999) LPS XXX, No. 1917.

[7] Berlin, J. (2008) Microsc. Microanal. 14 (Suppl 2) 110-111.

[8] Davis, J. M. (2009) Cement and Concrete Composites (submitted).

[9] Jolliff, B. L. (2008) LPS XXXIX, \#2519. [10] Vicenzi, E. P. (2008) LPS XXXIX, \#2335.

Table 1.

\begin{tabular}{|c|c|c|c|c|c|c|}
\hline & An ZAF & $\mathrm{En}_{80} \mathrm{ZAF}$ & K-el 50:50AF & 1 & 2 & 3 \\
\hline $\mathrm{Mg}$ & 1.414 & 1.486 & 0.0613 & 10.57 & 8.88 & 8.61 \\
\hline $\mathrm{Al}$ & 1.262 & 1.553 & 0.0768 & 8.15 & 9.45 & 10.45 \\
\hline $\mathrm{Si}$ & 1.322 & 1.318 & 0.1762 & 23.74 & 22.67 & 22.64 \\
\hline $\mathrm{Ca}$ & 1.089 & 1.090 & 0.0662 & 6.05 & 7.02 & 6.98 \\
\hline $\mathrm{Fe}$ & 1.191 & 1.180 & 0.0443 & 6.07 & 5.10 & 5.10 \\
\hline $\mathrm{O}$ & 3.045 & 1.874 & 0.1956 & 45.42 & 46.88 & 46.22 \\
\hline \multicolumn{7}{|c|}{$\begin{array}{l}\text { Key: An and En80 ZAF: Combined ZAF factor relative to pure elements for anorthite and } \mathrm{En}_{80} \mathrm{Fs}_{20} \text { pyroxene. } \\
K \text {-el } 50: 50 \mathrm{AF} \text { : Calculated k-ratios for a 50:50 area fraction using k-ratio for anorthite and } \mathrm{En}_{80} \text {. } \\
\text { 1. Ideal analysis for 50:50 AF anorthite }-\mathrm{En}_{80} \text { sample in weight percent using } \rho=2.76 \text { for anorthite and } \rho=3.81 \text { for } \mathrm{En}_{80} \text {. } \\
\text { 2. DBA analysis using k-ratios from 50:50 area fraction and } \mathrm{ZAF} \text { factors for anorthite and } \mathrm{En}_{80} \text {. } \\
\text { 3. Analysis obtained using k-ratios from 50:50 area fraction and assumption of homogeneous sample. }\end{array}$} \\
\hline
\end{tabular}

